PROCEEDINGS OF THE

AMERICAN MATHEMATICAL SOCIETY

Volume 132, Number 10, Pages 3035-3045

S 0002-9939(04)07337-X

Article electronically published on June 2, 2004

\title{
PUISEUX PARAMETRIC EQUATIONS OF ANALYTIC SETS
}

\author{
FUENSANTA AROCA
}

(Communicated by Ronald A. Fintushel)

\begin{abstract}
We prove the existence of local Puiseux-type parameterizations of complex analytic sets via Laurent series convergent on wedges. We describe the wedges in terms of the Newton polyhedron of a function vanishing on the discriminant locus of a projection. The existence of a local parameterization of quasi-ordinary singularities of complex analytic sets of any codimension will come as a consequence of our main result.
\end{abstract}

\section{INTRODUCTION}

The existence of local parametric equations at smooth points of an analytic set is a consequence of the implicit function theorem. At singular points the existence of a resolution of singularities [5] implies the existence of a finite system of sets of parametric equations covering a neighborhood of the singularity. However, desingularization theorems do not give much information about this system.

For a plane curve singularity, the Puiseux theorem asserts that we can find local parametric equations of the form $z_{1}=t^{k}, z_{2}=\phi(t)$, where $\phi$ is a convergent power series. A generalization of this result to non-planar curves can be found in [7].

The Abhyankar-Jung theorem [1] generalizes Puiseux's theorem to quasi-ordinary hypersurface singularities. (An $N$-dimensional analytic singularity, $(\mathcal{A}, a)$, is said to be quasi-ordinary if it admits a finite projection $p:(\mathcal{A}, a) \longrightarrow\left(\mathbb{C}^{N}, \underline{0}\right)$ whose discriminant locus is contained in a normal crossing divisor.)

$\mathrm{H}$. Hironaka introduced in 11 the notion of $\nu$-quasi-ordinary hypersurface singularity (a notion expressed in terms of the Newton polyhedron of the function defining the hypersurface). A proof of the Abhyankar-Jung theorem using this concept is given in [13]. A different proof can be found in [19].

Let $\mathcal{A} \subset \mathbb{C}^{N+M}, \underline{0} \in \mathcal{A}$, be an irreducible analytic set with $N=\operatorname{dim}_{\underline{0}}(\mathcal{A})$. When the discriminant locus of the projection $\left(z_{1}, \ldots, z_{N+M}\right) \mapsto\left(z_{1}, \ldots, z_{N}\right)$ is not contained in the coordinate hyperplanes (i.e., $\underline{0}$ is not a quasi-ordinary singularity), parametric equations of $\mathcal{A}$ around $\underline{0}$ of the form

$$
z_{i}=t_{i}{ }^{k}, i=1, \ldots, N, \quad z_{N+j}=\phi_{j}\left(t_{1}, \ldots, t_{N}\right), j=1, \ldots, M,
$$

where the $\phi_{j}$ 's are convergent power series do not always exist.

Received by the editors February 6, 2002 and, in revised form, May 19, 2003

2000 Mathematics Subject Classification. Primary 32S05, 32B10; Secondary 14M25.

Key words and phrases. Parameterization, wedges, quasi-ordinary singularities.

The author was supported first by Post-doctoral Grant of TMR Project Singularidades de Ecuaciones Diferenciales y Foliaciones at the University of Lisbon, and then by UNAM at Instituto de Matemáticas-Cuernavaca (Mexico). 
In order to state a result valid for non-quasi-ordinary singularities, it is then necessary to consider a ring bigger than the ring of ordinary convergent power series. For algebraic hypersurfaces, J. McDonald showed in [14] the existence of parametric equations of the form (1.1), where $\phi$ is a convergent Laurent series whose set of exponents is contained in a strongly convex polyhedral cone. With an additional hypothesis, P.D. González showed in 10] that the supporting cone can be chosen to be a cone of the Newton polytope of the discriminant of the polynomial defining the hypersurface with respect to $z_{N+1}$.

For a general analytic set $\mathcal{A} \subset \mathbb{C}^{N+M}, \underline{0} \in \mathcal{A}$, with $N=\operatorname{dim}_{\underline{0}}(\mathcal{A})$, we will show the existence of "local parametric equations" of the form (1.1), where the $\phi_{j}$ 's are convergent Laurent series with exponents contained in a strongly convex polyhedral cone. Due to the strong convexivity of the supporting cone, the common domain of convergence of the $\phi_{j}$ 's contains a non-empty open set. By analytic continuation, a set of such equations determines uniquely an irreducible branch of $\mathcal{A}$.

Series with exponents in a strongly convex cone appear naturally as Laurent series expansions of rational functions [9, Chapt. 6], as solutions of partial differential equations [4], and as solutions of holonomic systems [16]. Also, A. D. Bruno uses them in [6] to construct a method for solving non-linear differential equations.

We describe the supporting cones of the exponents of the parameterizations in terms of the discriminant locus of a projection. As a corollary we state a generalization of the Abhyankar-Jung theorem to quasi-ordinary complex analytic sets of any codimension.

I would like to thank J. M. Aroca, O. Neto and J. Snoussi for fruitful discussions and suggestions during the writing of this text. I thank also P. Popescu-Pampu for pointing out some mistakes in the first manuscript.

\section{Notation AND BAsiC DEFinitions}

Given $A \subset \mathbb{R}$ and $\alpha \in \mathbb{R}$ set $A_{\geq \alpha}:=\{a \in A ; a \geq \alpha\}$. Let $\tau: \mathbb{C}^{N} \longrightarrow\left(\mathbb{R}_{\geq 0}\right)^{N}$, and let $\log :\left(\mathbb{R}_{>0}\right)^{N} \longrightarrow \mathbb{R}^{N}$ be the maps defined by

$$
\begin{aligned}
\tau\left(z_{1}, z_{2}, \ldots, z_{N}\right) & :=\left(\left|z_{1}\right|,\left|z_{2}\right|, \ldots,\left|z_{N}\right|\right), \\
\log \left(x_{1}, x_{2}, \ldots, x_{N}\right) & :=\left(\log x_{1}, \log x_{2}, \ldots, \log x_{N}\right) .
\end{aligned}
$$

A set $\Omega \subset \mathbb{C}^{N}$ is called a Reinhardt set if $\tau^{-1}(\tau(\Omega))=\Omega$. A Reinhardt set $\Omega \subset\left(\mathbb{C}^{*}\right)^{N}\left(:=(\mathbb{C} \backslash\{0\})^{N}\right)$ is said to be logarithmically convex if the set $\log (\tau(\Omega))$ is convex.

Let $k$ be a natural number, and let $\xi_{k}: \mathbb{C}^{N} \longrightarrow \mathbb{C}^{N}$ be defined by

$$
\xi_{k}\left(z_{1}, z_{2}, \ldots, z_{N}\right):=\left(z_{1}{ }^{k}, z_{2}{ }^{k}, \ldots, z_{N}{ }^{k}\right) .
$$

Given $\Omega \subset \mathbb{C}^{N}$, set $\sqrt[k]{\Omega}:=\xi_{k}^{-1}(\Omega)$ and $|\sqrt[k]{\Omega}|:=\tau\left(\xi_{k}^{-1}(\Omega)\right)$.

Given $\mathcal{G}=\left\{u^{(1)}, u^{(2)}, \ldots, u^{(M)}\right\} \subset \mathbb{Z}^{N}$, the convex polyhedral cone generated by $\mathcal{G}$ is the convex set $\langle\mathcal{G}\rangle:=\left\{\lambda_{1} u^{(1)}+\lambda_{2} u^{(2)}+\cdots+\lambda_{M} u^{(M)} ; \lambda_{j} \in \mathbb{R}_{\geq 0}\right\}$. A cone $\sigma$ is rational if there exists $\mathcal{G} \subset \mathbb{Z}^{N}$ such that $\sigma=\langle\mathcal{G}\rangle$. By cone we will mean convex polyhedral cone. A cone is said to be strongly convex if it does not contain any proper linear subspace.

For $\vartheta \in \mathbb{R}^{N} \backslash\{\underline{0}\}$ and $a \in \mathbb{R}$, we will denote by $H^{+}(\vartheta ; a)$ the affine half-space $\left\{u \in \mathbb{R}^{N} ; u \cdot \vartheta \geq a\right\}$. 
Given $\varrho=\left(\varrho_{1}, \ldots, \varrho_{N}\right) \in\left(\mathbb{R}_{>0}\right)^{N}$, we set

$$
\overline{\mathbb{D}}_{\varrho}:=\left\{z=\left(z_{1}, \ldots, z_{N}\right) \in \mathbb{C}^{N} ; \quad\left|z_{i}\right| \leq \varrho_{i}, i=1, \ldots, N\right\}
$$

and $\overline{\mathbb{D}}_{\varrho}^{*}:=\overline{\mathbb{D}}_{\varrho} \cap\left(\mathbb{C}^{*}\right)^{N}$, where $\mathbb{C}^{*}:=\mathbb{C} \backslash\{0\}$.

Given $u=\left(I_{1}, I_{2}, \ldots, I_{N}\right) \in \mathbb{Z}^{N}$ and $z=\left(z_{1}, z_{2}, \ldots, z_{N}\right)$ we set

$$
z^{u}:=z_{1}^{I_{1}} z_{2}^{I_{2}} \cdots z_{N}{ }^{I_{N}} .
$$

Given an $M$-tuple of vectors $\mathcal{G}=\left\{u^{(1)}, \ldots, u^{(M)}\right\} \subset \mathbb{Z}^{N}$, set $\Phi_{\mathcal{G}}:\left(\mathbb{C}^{*}\right)^{N} \longrightarrow$ $\left(\mathbb{C}^{*}\right)^{M}$ to be the map defined by

$$
\Phi_{\mathcal{G}}(z):=\left(z^{u^{(1)}}, z^{u^{(2)}}, \ldots, z^{u^{(M)}}\right) .
$$

Given a Laurent series $s=\sum_{I \in \mathbb{Z}^{N}} a_{I} z^{I}$, we call $\mathcal{E}(s):=\left\{I \in \mathbb{Z}^{N} ; a_{I} \neq 0\right\}$ the set of exponents of $s$.

Let $\delta:\left(\mathbb{C}^{N}, \underline{0}\right) \longrightarrow(\mathbb{C}, 0)$ be a germ of an analytic function. The set of exponents of its Taylor series development is contained in $\left(\mathbb{R}_{\geq 0}\right)^{N}$. The Newton polyhedron of $\delta, \operatorname{NP}(\delta)$, is the convex hull of the set $\mathcal{E}(\delta)+\left(\mathbb{R}_{\geq 0}\right)^{N}$. See Figure 1.
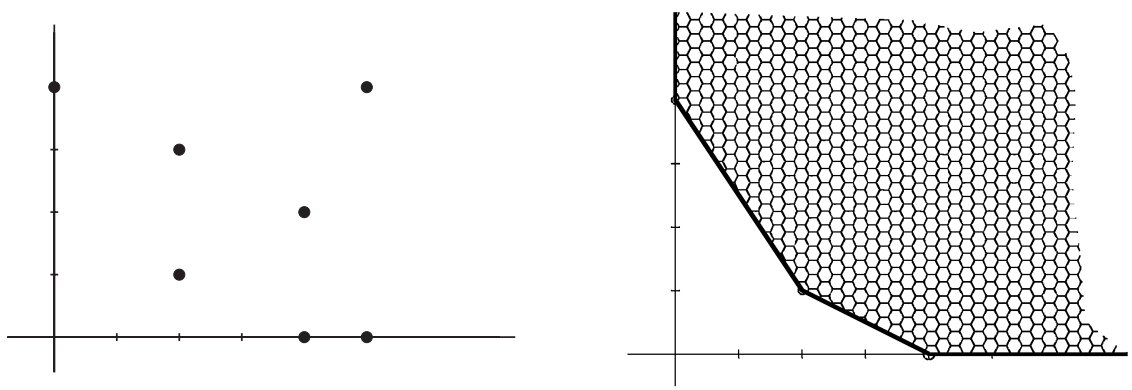

Figure 1. The dots on the left are the set of exponents of $f:=$ $\left(z_{1}^{4}+z_{1}{ }^{5}+z_{2}{ }^{4}+z_{1}{ }^{2} z_{2}+z_{1}{ }^{4} z_{2}{ }^{2}+z_{1}{ }^{2} z_{2}{ }^{3}+z_{1}{ }^{5} z_{2}{ }^{4}\right)$. On the right is the Newton polyhedron $\mathrm{NP}(f)$.

Let $A$ be a vertex of the Newton polyhedron of an analytic function $\delta$. The set of vectors $v \in \mathbb{R}^{N}$ such that $(A+\lambda v) \in \operatorname{NP}(\delta)$ for some positive real number $\lambda$ is a cone $\sigma$. We will say that $\sigma$ is the cone of the Newton polyhedron of $\delta$, associated to the vertex $A$. The cone $\sigma$ is just the cone spanned by the faces of $\operatorname{NP}(\delta)$ that contain $A$. Cones of the Newton polyhedron of an analytic function are always strongly convex and contain the non-negative orthant $\left(\mathbb{R}_{\geq 0}\right)^{N}$. See Figure 2 .


Figure 2. Cones associated to the vertices of $\mathrm{NP}(f)$. 


\section{WEDGES AND CONES}

We introduce the notion of $\sigma$-wedge, which is the analog for series with exponents in a cone $\sigma$, to polydiscs for series with non-negative exponents.

Suppose $s$ is a Laurent series convergent at a point $p \in\left(\mathbb{C}^{*}\right)^{N}$; then $s$ is also convergent at any point $z \in\left(\mathbb{C}^{*}\right)^{N}$ with $\tau(z)^{I}<\tau(p)^{I}$ for all $I \in \mathcal{E}(s)$.

Definition 3.1. Let $\sigma \subset \mathbb{R}^{N}$ be a cone. For $\varrho \in\left(\mathbb{R}_{>0}\right)^{N}$, the $\sigma$-wedge of polyradius $\varrho$ is the set

$$
\mathrm{W}(\sigma, \varrho):=\left\{z \in\left(\mathbb{C}^{*}\right)^{N} ; \tau(z)^{u} \leq \varrho^{u}, \forall u \in \sigma \cap \mathbb{Z}^{N}\right\} .
$$

It is clear that $\sigma$-wedges are Reinhardt sets.

Example 3.2. Let $\mathcal{C}$ be the canonical basis of $\mathbb{R}^{N}$. For any $\varrho \in\left(\mathbb{R}_{>0}\right)^{N}$, the $\langle\mathcal{C}\rangle$-wedge of polyradius $\varrho$ is $\overline{\mathbb{D}}_{\varrho}^{*}$.
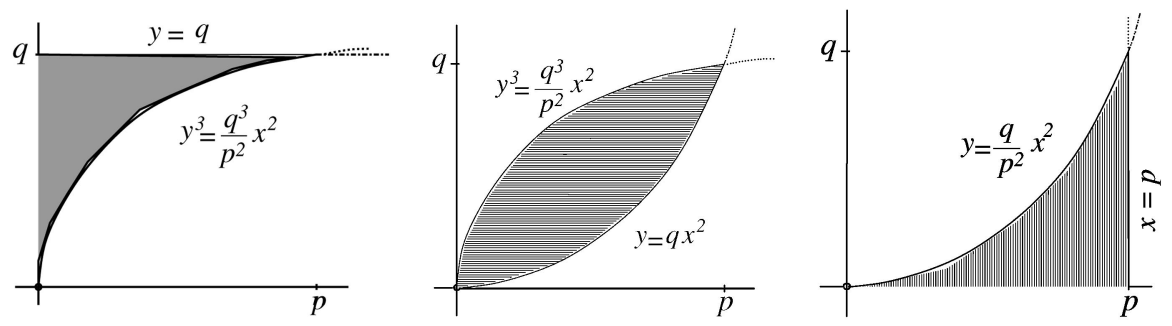

FiguRE 3. The image under $\tau$ of $\sigma$-wedges of polyradius $(p, q)$, where the cones $\sigma$ are those of $\mathrm{NP}(f)$.

Let $\sigma$ be the cone generated by $\mathcal{G}=\left\{u^{(1)}, \ldots, u^{(M)}\right\} \subset \mathbb{Z}^{N}$. For any $\varrho \in\left(\mathbb{R}_{>0}\right)^{N}$ we have:

$$
\begin{aligned}
& \mathrm{W}(\sigma, \varrho)=\left\{z \in\left(\mathbb{C}^{*}\right)^{N} ; \tau(z)^{u^{(i)}} \leq \varrho^{u^{(i)}}, \forall i=1, \ldots, M\right\} ; \\
& \mathrm{W}(\sigma, \varrho)=\Phi_{\mathcal{G}}^{-1}\left(\overline{\mathbb{D}}_{\Phi_{\mathcal{G}}(\varrho)}^{*}\right) ; \\
& \text { if } \sigma \subset \sigma^{\prime} \text {, then } \mathrm{W}\left(\sigma^{\prime}, \varrho\right) \subset \mathrm{W}(\sigma, \varrho) \text {; } \\
& \sqrt[k]{\mathrm{W}(\sigma, \varrho)}=\mathrm{W}(\sigma,|\sqrt[k]{\varrho}|) ; \\
& \log (\tau(\mathrm{W}(\sigma, \varrho)))=\bigcap_{u \in \sigma \cap \mathbb{Z}^{N}} H^{+}\left(-u ;-\log \varrho^{u}\right) .
\end{aligned}
$$

Proposition 3.3. Let $\sigma$ be a rational cone. A $\sigma$-wedge is a connected set with connected interior.

Proof. For any $\varrho \in\left(\mathbb{R}_{>0}\right)^{N}, \log (\tau(\mathrm{W}(\sigma, \varrho)))$ is the intersection of affine half hyperplanes (see (3.5)) and therefore a connected set of connected interior. The result follows from the fact that Log is a homeomorphism and $\tau$ is a projection with connected fiber.

Proposition 3.4. Let $\sigma$ be a strongly convex rational cone. For any pair $\varrho, \varrho^{\prime} \in$ $\left(\mathbb{R}_{>0}\right)^{N}$, the interior of $\mathrm{W}(\sigma, \varrho) \cap \mathrm{W}\left(\sigma, \varrho^{\prime}\right)$ is not empty. 
Proof. Suppose $\sigma$ is strongly convex, and let $\vartheta$ be a vector of integer coordinates such that $\sigma \subset H^{+}(\vartheta ; 0)$ and $\sigma \cap \vartheta^{\perp}=\{\underline{0}\}\left[\underline{8}\right.$, 1.2 (13)]. Each $u \in \sigma \cap \mathbb{Z}^{N} \backslash\{\underline{0}\}$ can be written in a unique way as a sum $u=v_{u}+\lambda_{u} \vartheta$ where $v_{u} \in \vartheta^{\perp}$ and $\lambda_{u} \in \mathbb{Q}_{>0}$.

Let $\mathcal{G} \subset \mathbb{Z}^{N}$ be a finite set of generators of $\sigma$. Set $\eta=\frac{1}{2} \min _{u \in \mathcal{G}}\left\{\min \left\{\varrho^{\frac{1}{\lambda_{u} u}}, \varrho^{\prime \frac{1}{\lambda_{u} u}}\right\}\right\}$; since the $\lambda_{u}$ 's are positive rational numbers, $\eta>0$.

Let $\mathcal{B}=\left\{e^{(1)}, \ldots, e^{(N-1)}, \vartheta\right\} \subset \mathbb{Z}^{N}$ be an orthogonal basis of $\mathbb{R}^{N}$. Let $\Phi_{\mathcal{B}}$ be as in (2.1); since $\mathcal{B}$ is a basis, $\Phi_{\mathcal{B}}$ is an automorphism of $\mathbb{C}^{* N}$. Let $\omega=\Phi_{\mathcal{B}}{ }^{-1}(1, \ldots, 1, \eta)$. For each $u \in \sigma$,

$$
\tau(\omega)^{u}=\tau(\omega)^{v_{u}} \tau(\omega)^{\lambda_{u} \vartheta}=\left|\omega^{v_{u}}\right|\left|\omega^{\vartheta}\right|^{\lambda_{u}}=1 \eta^{\lambda_{u}} \leq \frac{1}{2} \min \left\{\varrho^{u}, \varrho^{\prime u}\right\}<\min \left\{\varrho^{u}, \varrho^{\prime u}\right\} .
$$

Hence $\omega$ belongs to the interior of $W(\sigma, \varrho) \cap W\left(\sigma, \varrho^{\prime}\right)$.

Remark 3.5. Suppose that $\sigma$ is not strongly convex. Then there exists $\vartheta \in \mathbb{Z}^{n} \backslash\{\underline{0}\}$ such that $\langle\vartheta,-\vartheta\rangle \subset \sigma$. For any $\varrho \in\left(\mathbb{R}_{>0}\right)^{N}$,

$$
\begin{aligned}
\mathrm{W}(\sigma, \varrho) \stackrel{\sqrt{3.3}}{\complement} \mathrm{W}(\langle\vartheta,-\vartheta\rangle, \varrho) \stackrel{\sqrt{3.1}}{=}\left\{z \in\left(\mathbb{C}^{*}\right)^{N} ; \tau(z)^{\vartheta}\right. & \left.\leq \varrho^{\vartheta}, \tau(z)^{-\vartheta} \leq \varrho^{-\vartheta}\right\} \\
& =\left\{z \in\left(\mathbb{C}^{*}\right)^{N} ; \tau(z)^{\vartheta}=\varrho^{\vartheta}\right\}
\end{aligned}
$$

(so its interior is empty).

Proposition 3.6. Let $\sigma$ be a rational cone. A $\sigma$-wedge is a logarithmically convex Reinhardt set. If $\sigma$ is strongly convex, then its interior is a non-empty logarithmically convex Reinhardt domain.

Proof. For any $\varrho \in\left(\mathbb{R}_{>0}\right)^{N}, \log (\tau(\mathrm{W}(\sigma, \varrho)))$ is the intersection of affine half hyperplanes (see (3.5)) and therefore a convex set. If $\sigma$ is strongly convex, then its interior is connected (Proposition 3.3) and non-empty (Proposition 3.4).

\section{LAURENT SERIES DEVELOPMENT OVER A $\sigma$-WEDGE}

A holomorphic function $\varphi$, defined on a Reinhardt domain $\Omega$, has a (unique) Laurent series expansion that converges uniformly to $\varphi$ on compact subsets of $\Omega$ [2, I.6].

The Taylor development of a function, holomorphic on a disc centered at the origin, is a series with exponents in the non-negative orthant. In this section we will see a similar result for $\sigma$-wedges and series with exponents in the cone $\sigma$.

Definition 4.1. Let $\sigma$ be a cone. We will say that a set $\Omega \subset\left(\mathbb{C}^{*}\right)^{N}$ is $\sigma$-complete if for any $z \in \Omega$ the $\sigma$-wedge of polyradius $\tau(z)$ is contained in $\Omega$.

A $\sigma$-wedge is $\sigma$-complete. A $\sigma$-complete set is a Reinhardt set. If $\sigma$ is strongly convex, from Propositions 3.3 and 3.4 it follows that a $\sigma$-complete set is connected with connected interior.

Remark 4.2. The domain of convergence of a Laurent series with exponents in a cone $\sigma$ is $\sigma$-complete.

Remark 4.3. As a consequence of (3.4) we have that, if $\Omega$ is $\sigma$-complete and $k$ is a natural number, then $\sqrt[k]{\Omega}$ is $\sigma$-complete. 
Lemma 4.4. Let $\Omega$ be a Reinhardt domain, let $\varphi: \Omega \longrightarrow \mathbb{C}$ be a bounded holomorphic function, and take $\vartheta \in \mathbb{Z}^{N} \backslash\{\underline{0}\}$. If $\Omega$ is $H^{+}(\vartheta ; 0)$-complete, then the set of exponents of the Laurent series expansion of $\varphi$ is contained in $H^{+}(\vartheta ; 0)$.

Proof. Let $\mathcal{B}=\left\{e^{(1)}, \ldots, e^{(N-1)}, \vartheta\right\} \subset \mathbb{Z}^{N}$ be an orthogonal basis of $\mathbb{R}^{N}$. We have $H^{+}(\vartheta ; 0)=\left\langle e^{(1)}, \ldots, e^{(N-1)},-e^{(1)}, \ldots,-e^{(N-1)}, \vartheta\right\rangle$.

For each $I \in \mathbb{Z}^{N}$ let $I_{\mathcal{B}}$ be the coordinates of $I$ in the basis $\mathcal{B}$. Since $\mathcal{B} \subset \mathbb{Z}^{N}$, there exists a natural number $d$ such that $d I_{\mathcal{B}} \in \mathbb{Z}^{N}$ for any $I \in \mathbb{Z}^{N}$.

Let $s=\sum_{I \in \mathbb{Z}^{N}} a_{I} z^{I}$ be the Laurent series expansion of $\varphi$, and set

$$
t:=\sum_{I \in \mathbb{Z}^{N}} a_{I} y^{d I_{\mathcal{B}}}, \quad \text { where } \quad y=\left(y_{1}, \ldots, y_{N}\right) .
$$

For any $z \in \sqrt[d]{\Omega}, t\left(\Phi_{\mathcal{B}}(z)\right)=s\left(z^{d}\right)$. Hence $t$ is convergent and bounded in $\Phi_{\mathcal{B}}(\sqrt[d]{\Omega})$.

From the way $t$ has been defined:

S) $\mathcal{E}(s) \subset H^{+}(\vartheta ; 0)$ if and only if $\mathcal{E}(t) \subset H^{+}((0, \ldots, 0,1) ; 0)$.

Let $\pi$ be the map defined by $\pi\left(y_{1}, \ldots, y_{N}\right):=\left(y_{1}, \ldots, y_{N-1}\right)$, and let us rewrite the series $t$ in the form

$$
t=\sum_{j \in \mathbb{Z}} g_{j}(\pi(y)) y_{N}{ }^{j}, \quad \text { where } \quad g_{j}=\sum_{(0, \ldots, 0,1) \cdot d I_{\mathcal{B}}=j} a_{I} x^{d \pi\left(I_{\mathcal{B}}\right)}
$$

for $x=\left(x_{1}, \ldots, x_{N-1}\right)$.

Now statement $\mathbf{S}$ ) becomes:

$\left.\mathbf{S}^{\prime}\right) \mathcal{E}(s) \subset H^{+}(\vartheta ; 0)$ if and only if $g_{j}(x) \equiv 0$ for all $j<0$.

For any $\varrho \in \tau(\sqrt[d]{\Omega})$, by Remark 4.3 the wedge $\mathrm{W}\left(H^{+}(\vartheta, 0), \varrho\right)$ is contained in $\sqrt[d]{\Omega}$. So, $\Phi_{\mathcal{B}}\left(\mathrm{W}\left(H^{+}(\vartheta, 0), \varrho\right)\right) \subset \Phi_{\mathcal{B}}(\sqrt[d]{\Omega})$. Hence $t$ is convergent and bounded on:

$$
\Phi_{\mathcal{B}}\left(\mathrm{W}\left(H^{+}(\vartheta, 0), \varrho\right)\right)=\left\{y \in\left(\mathbb{C}^{*}\right)^{N} ; \quad\left|y_{N}\right| \leq \varrho^{\vartheta},\left|y_{i}\right|=\varrho^{e^{(i)}}, i=1, \ldots, N-1\right\} .
$$

For any $z \in \sqrt[d]{\Omega}$, the series in one variable,

$$
t_{z}\left(y_{N}\right):=t\left(\pi\left(\Phi_{\mathcal{B}}(z)\right), y_{N}\right)=\sum_{j \in \mathbb{Z}} g_{j}\left(\pi\left(\Phi_{\mathcal{B}}(z)\right)\right) y_{N}{ }^{j}
$$

is convergent and bounded on the punctured disc $\mathbb{D}_{\tau(z)^{\vartheta}}^{*}$. Then, there exists a unique function, holomorphic on $\mathbb{D}_{\tau(z)^{\vartheta}}$, that extends $t_{z}$. Therefore $t_{z}$ is necessarily the Taylor development of that function and, as a consequence, it cannot have negative exponents.

We have seen that, for $j<0, g_{j}(x)=0$ for all $x \in \pi\left(\Phi_{\mathcal{B}}(\sqrt[d]{\Omega})\right)$. Since $\pi\left(\Phi_{\mathcal{B}}(\sqrt[d]{\Omega})\right)$ is a non-empty open subset of $\mathbb{C}^{N-1}, g_{j} \equiv 0$.

Proposition 4.5. Let $\sigma$ be a cone, and let $\Omega \subset\left(\mathbb{C}^{*}\right)^{N}$ be a $\sigma$-complete Reinhardt domain. If $\varphi$ is a bounded holomorphic function on $\Omega$, and $s$ is its Laurent series expansion in $\Omega$, then the set of exponents of $s$ is contained in $\sigma$.

Proof. Let $\vartheta \in \mathbb{Z}^{N} \backslash\{\underline{0}\}$ be such that $\sigma \subset H^{+}(\vartheta ; 0)$. By (3.3), $\Omega$ is $H^{+}(\vartheta ; 0)$ complete. So (Lemma 4.4) $\mathcal{E}(s) \subset H^{+}(\vartheta ; 0)$, and the result follows from the fact that

$$
\sigma=\bigcap_{\vartheta \in \mathbb{Z}^{N}, \sigma \subset H^{+}(\vartheta ; 0)} H^{+}(\vartheta ; 0),
$$

for any cone $\sigma$. 


\section{Cones of A POlyhedron And Wedges NOT INTERSECTING THE ZERO-LOCUS}

Proposition 5.1. Let $\delta:\left(\mathbb{C}^{N}, \underline{0}\right) \longrightarrow(\mathbb{C}, 0)$ be a germ of an analytic function, and let $\sigma$ be a cone of the Newton polyhedron of $\delta$. There exists a $\sigma$-wedge that does not intersect the zero locus of $\delta$.

Proof. Let $\mathcal{T}(\delta)=\sum_{I \in \mathcal{E}(\delta)} b_{I} z^{I}$ be the Taylor development of $\delta$, and let $\Omega$ be its domain of convergence. Let $A$ be the vertex of $\sigma$ in $\mathrm{NP}(\delta)$. The meromorphic function $f=z^{-A} \delta$ is holomorphic on $\Omega^{*}=\Omega \cap\left(\mathbb{C}^{*}\right)^{N}$, and, outside the coordinate hyperplanes, the zero locus of $\delta$ coincides with the zero locus of $f$. We will find a $\sigma$-wedge where $f$ does not vanish.

Notice that the Laurent series development of $f$ on $\Omega^{*}$,

$$
s=\frac{1}{z^{A}} \mathcal{T}(\delta)=\sum_{I-A \in \sigma \cap \mathbb{Z}^{N}} b_{I} z^{I-A}=\sum_{I \in \sigma \cap \mathbb{Z}^{N}} a_{I} z^{I}, \quad a_{I}=b_{I+A},
$$

is a series with exponents in $\sigma$.

Let $\mathcal{G}=\left\{u^{(1)}, u^{(2)}, \ldots, u^{(M)}\right\}$ be a set of generators of the semigroup $\sigma \cap \mathbb{Z}^{N}$ (Gordan's lemma [8 1.2, Prop. 1]). For each $I \in \mathcal{E}(s)$, let $I_{\mathcal{G}}$ be an $M$-tuple of natural numbers $I_{\mathcal{G}}=\left(I_{\mathcal{G}}^{1}, \ldots, I_{\mathcal{G}}^{M}\right)$ such that $I=I_{\mathcal{G}}^{1} u^{(1)}+I_{\mathcal{G}}^{2} u^{(2)}+\cdots+I_{\mathcal{G}}^{M} u^{(M)}$.

The series in the variables $y=\left(y_{1}, y_{2}, \ldots, y_{M}\right)$ defined by

$$
t(y)=\sum_{I \in \mathcal{E}(\delta)} a_{I} y^{I_{\mathcal{G}}}
$$

is a series with positive exponents.

Let $\Phi_{\mathcal{G}}$ be as in (2.1). For any $z \in\left(\mathbb{C}^{*}\right)^{N}$ contained in the domain of convergence of $s$, we have that

$$
t\left(\Phi_{\mathcal{G}}(z)\right)=s(z) .
$$

In particular, $t$ is convergent in $\Phi_{\mathcal{G}}(z)$. Now, since $t$ has positive exponents, $t$ converges in $\overline{\mathbb{D}}_{\Phi_{\mathcal{G}}(\tau(z))}$ (the closed polydisc of polyradius $\tau\left(\Phi_{\mathcal{G}}(z)\right)$ ).

The series $t$ is convergent, does not have negative exponents, and $t(\underline{0})=b_{\underline{0}}=$ $a_{A} \neq 0$. So there exists $\varsigma \in\left(\mathbb{R}_{>0}\right)^{M}$ such that $t(y) \neq 0$ for any $y \in \overline{\mathbb{D}}_{\varsigma}^{*}$. Take $\varrho \in \tau\left(\Phi_{\mathcal{G}}^{-1}\left(\overline{\mathbb{D}}_{\varsigma}^{*}\right)\right)$. By (3.2), the $\sigma$-wedge of polyradius $\varrho$ has the desired property.

\section{Coverings of LOGarithmically CONVEX Reinhardt domains}

In this section we extend the well-known fact that coverings of the punctured disc are isomorphic to some covering $t \mapsto t^{k}$.

Lemma 6.1. The fundamental group of a logarithmically convex Reinhardt domain $\Omega \subset \mathbb{C}^{* N}$ is isomorphic to $\mathbb{Z}^{N}$.

Proof. The projection $\tau: \Omega \longrightarrow \tau(\Omega)$ is a fiber bundle with fiber an $N$-dimensional torus. $\log (\tau(\Omega))$ is convex and then contractible. Since $\log$ is a homeomorphism, $\tau(\Omega)$ is also contractible. Then (see, for example, $[17, \S 11.6]) \Omega$ is homeomorphic to $\mathbb{T}^{N} \times \tau(\Omega)$. Again as a consequence of the contractibility of $\tau(\Omega)$ we have the result.

Proposition 6.2. Let $\mathcal{A}$ be a complex analytic set. Let $p: \mathcal{A} \longrightarrow \mathbb{C}^{N}$ be a finite morphism. Let $\Omega \subset\left(\mathbb{C}^{*}\right)^{N}$ be a logarithmically convex Reinhardt domain contained in $\mathrm{p}(\mathcal{A})$ such that $\mathrm{p}: \mathcal{A} \cap \mathrm{p}^{-1}(\Omega) \longrightarrow \Omega$ is locally biholomorphic. Then for each 
connected component $\mathrm{C}$ of $\mathrm{p}^{-1}(\Omega)$ there exists a natural number $k$ and a morphism $\varphi: \sqrt[k]{\Omega} \longrightarrow \mathrm{C}$ such that $\varphi(\sqrt[k]{\Omega})=\mathrm{C}$ and $\mathrm{p} \circ \varphi=\xi_{k}$.

Proof. Let $\mathrm{C}$ be a connected component of $\mathrm{p}^{-1}(\Omega)$, and let $k$ be the cardinal of the generic fiber of $\left.\mathrm{p}\right|_{\mathrm{C}}$. Since both $\left.\mathrm{p}\right|_{\mathrm{C}}$ and $\left.\xi_{k}\right|_{\mathrm{C}}$ are locally biholomorphic, the pairs $(\mathrm{C}, \mathrm{p})$ and $\left(\sqrt[k]{\Omega}, \xi_{k}\right)$ are, respectively, a $k$-sheeted and a $k^{N}$-sheeted covering of $\Omega$ :

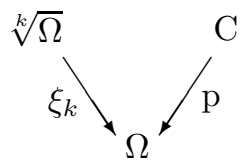

Choose a point $z_{0} \in \Omega$, a point $z_{1} \in \xi_{k}^{-1}\left(z_{0}\right)$, and a point $P$ in $\mathrm{p}^{-1}\left(z_{0}\right) \cap \mathrm{C}$. Consider the induced monomorphisms on the fundamental groups:

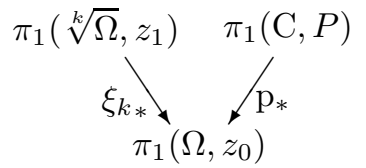

An element $\gamma \in \pi_{1}\left(\Omega, z_{0}\right)$ is in the subgroup $\xi_{k_{*}} \pi_{1}\left(\Omega, z_{0}\right)$ if and only if $\gamma=\alpha^{k}$ for some $\alpha \in \pi_{1}\left(\Omega, z_{0}\right)$.

On the other hand, the index of $\mathrm{p}_{*}\left(\pi_{1}(\mathrm{C}, P)\right)$ in $\pi_{1}\left(\Omega, z_{0}\right)$ is equal to $k$ (see, for example, [15, V $\S 7])$. Since $\pi_{1}\left(\Omega, z_{0}\right)$ is abelian, the cosets of $\mathrm{p}_{*}\left(\pi_{1}(\mathrm{C}, P)\right)$ in $\pi_{1}\left(\Omega, z_{0}\right)$ form a group of order $k$. Therefore, for any $\alpha \in \pi_{1}\left(\Omega, z_{0}\right)$ the element $\alpha^{k}$ belongs to $\mathrm{p}_{*}\left(\pi_{1}(\mathrm{C}, P)\right)$. Then $\xi_{k_{*}}\left(\pi_{1}\left(\sqrt[k]{\Omega}, z_{1}\right)\right) \subset \mathrm{p}_{*}\left(\pi_{1}(\mathrm{C}, P)\right)$.

The lifting lemma [15] Theorem V.5.1] ensures the existence of a (unique) map $\varphi$, such that $\varphi\left(z_{1}\right)=P$ and the diagram

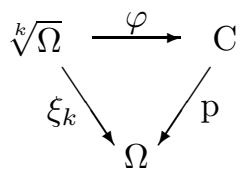

commutes.

Remark 6.3. In Proposition 6.2, $k$ can be chosen to be the degree of the covering $\mathrm{p}: \mathrm{C} \longrightarrow \Omega$. Since for each $P \in \mathrm{p}^{-1}\left(z_{0}\right) \cap \mathrm{C}$ there exists a unique $\varphi$ such that $\varphi\left(z_{1}\right)=P$, there exist $k$ different $\varphi$ 's.

\section{THE THEOREM}

Let $\mathcal{A}$ be an analytic set, and assume that $\underline{0} \in \mathcal{A} \subset \mathbb{C}^{N+M}$, where $N=\operatorname{dim}_{\underline{0}} \mathcal{A}$ is the dimension of $\mathcal{A}$ at $\underline{0}$. There exists a linear projection $p: \mathbb{C}^{N+M} \longrightarrow \mathbb{C}^{N}$, and a neighborhood $U$ of $\underline{0}$ such that the restriction of $p$ to $\mathcal{A} \cap U$ is a finite morphism. Moreover, the set of such $p$ is open and everywhere dense in the space of linear projections.

Let $\mathrm{p}: \mathcal{A} \cap U \longrightarrow \mathbb{C}^{N}$ be a finite morphism. Then $\mathrm{p}(\mathcal{A} \cap U)$ is a neighborhood of $\mathrm{p}(\underline{0})$ and there exists an analytic set $\Delta \subset \mathbb{C}^{N}$, with $\operatorname{dim}_{\mathrm{p}(\underline{0})} \Delta<N$, such that $\mathrm{p}: \mathcal{A} \cap U \backslash \mathrm{p}^{-1}(\Delta) \longrightarrow \mathrm{p}(\mathcal{A} \cap U) \backslash \Delta$ is locally biholomorphic [7, 3.7]. The smallest set $\Delta$ with this property will be called the discriminant locus of $\mathrm{p}$. 
Theorem 7.1. Let $\mathcal{A}$ be an analytic subset of $\mathbb{C}^{N+M}, \underline{0} \in \mathcal{A}, \operatorname{dim}_{\underline{0}}(\mathcal{A})=N$. Let $U$ be a neighborhood of $\underline{0}$, and let $\pi$ be the restriction to $\mathcal{A} \cap U$ of the projection $\left(z_{1}, z_{2}, \ldots, z_{N+M}\right) \mapsto\left(z_{1}, z_{2}, \ldots, z_{N}\right)$. Assume $\pi$ is a finite morphism. Let $\delta$ be an analytic function vanishing on the discriminant locus of $\pi$.

For each cone $\sigma$ of the Newton polyhedron of $\delta$, there exist $k \in \mathbb{N}$ and an $M$-tuple of convergent Laurent series, $\left(s_{1}, \ldots, s_{M}\right)$, such that

$$
\mathcal{E}\left(s_{i}\right) \subset \sigma, \quad i=1, \ldots, M,
$$

and

$$
f\left(z_{1}^{k}, \ldots, z_{N}^{k}, s_{1}, \ldots, s_{M}\right)=0
$$

for any $f$ vanishing on $\mathcal{A}$, and any $z$ in the domain of convergence of the $s_{i}$.

Proof. Let us choose $\varrho_{1}$ small enough so that $\overline{\mathbb{D}}_{\varrho_{1}}$ is contained in $\pi(\mathcal{A} \cap U)$ and so that $\pi^{-1}\left(\mathbb{D}_{\varrho_{1}}\right)$ is a bounded set of $\mathbb{C}^{N+M}$. Since $\sigma$ contains the non-negative orthant, by (3.3), $\mathrm{W}\left(\sigma, \varrho_{1}\right) \subset \pi(\mathcal{A} \cap U)$.

By Proposition 5.1 we can find an $N$-tuple of positive real numbers, $\varrho_{2}$, such that $\mathrm{W}\left(\sigma, \varrho_{2}\right)$ does not intersect the zero locus of $\delta$. Since $\delta$ vanishes on the discriminant locus of $\pi, \mathrm{W}\left(\sigma, \varrho_{2}\right)$ does not intersect the discriminant locus, and $\pi$ restricted to $\mathrm{W}\left(\sigma, \varrho_{2}\right)$ is locally biholomorphic.

Since $\sigma$ is strongly convex, the intersection $\mathrm{W}\left(\sigma, \varrho_{1}\right) \cap \mathrm{W}\left(\sigma, \varrho_{2}\right)$ is non-empty (Proposition 3.4). Take $\varrho \in \tau\left(\mathrm{W}\left(\sigma, \varrho_{1}\right) \cap \mathrm{W}\left(\sigma, \varrho_{2}\right)\right)$. Let $\Omega$ be the interior of $\mathrm{W}(\sigma, \varrho)$.

Since $\sigma$ is strongly convex, by Proposition 3.6 , $\Omega$ is a non-empty logarithmically convex Reinhardt domain.

Let $\mathrm{C}$ be a connected component of $\pi^{-1}(\Omega)$. Then, by Proposition 6.2, there exists a natural number $k$ and a morphism $\varphi: \sqrt[k]{\Omega} \longrightarrow \mathrm{C}$, such that $\pi \circ \varphi=\xi_{k}$. We can express $\varphi$ as

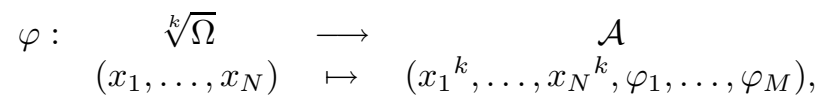

where $\varphi_{i}: \sqrt[k]{\Omega} \longrightarrow \mathbb{C}$ is a holomorphic function for $i \in\{1, \ldots, M\}$. Moreover, since $\varphi(\sqrt[k]{\Omega}) \subset \mathcal{A} \cap U$, each $\varphi_{i}$ is bounded.

Since $\sqrt[k]{\Omega}$ is $\sigma$-complete, by Proposition 4.5 we have that for each $i \in\{1, \ldots, M\}$, there is a Laurent series $s_{i}$ that converges uniformly to $\varphi_{i}$ in compact subsets of $\sqrt[k]{\Omega}$ and such that $\mathcal{E}\left(s_{i}\right) \subset \sigma$.

Now, for any $z$ in the domain of convergence of the $s_{i}$ 's, the point of $\mathbb{C}^{N+M}$, $P=\left(z_{1}{ }^{k}, \ldots, z_{N}{ }^{k}, s_{1}(z), \ldots, s_{M}(z)\right)$ is in $\mathcal{A}$ (or in an analytic extension of $\mathcal{A}$ ) and, therefore, any function $f$ vanishing on $\mathcal{A}$ also vanishes on $P$.

Remark 7.2. Taking as $\mathrm{C}$ the different connected components of $\pi^{-1}(\Omega)$, we may in fact find $k_{1}, \ldots, k_{r}$ natural numbers with $\sum_{i=1}^{r} k_{i}$ equal to the degree of $\pi$ and, for each $i \in\{1, \ldots, r\}, k_{i}$ different $M$-tuples of series. In other words, we have seen that if $d$ is the degree of $\pi$, there are $d M$-tuples of Puiseux series such that $f\left(z_{1}, \ldots, z_{N}, s_{1}, \ldots, s_{M}\right)=0$ for any $f \in I(\mathcal{A})$.

Corollary 7.3 (Abhyankar-Jung). Let $\underline{0}$ be a quasi-ordinary singularity of a complex analytic set $\mathcal{A} \subset \mathbb{C}^{N+M}, \operatorname{dim}_{\underline{\underline{0}}}(\mathcal{A})=N$. Then there exists a natural number 
$k$, and $M$ convergent power series $\phi_{j}, j=1, \ldots, M$, such that

$$
z_{i}=t_{i}{ }^{k}, i=1, \ldots, N, \quad z_{N+j}=\phi_{j}\left(t_{1}, \ldots, t_{N}\right), j=1, \ldots, M,
$$

are parametric equations of $\mathcal{A}$ about $\underline{0}$.

Proof. By definition of a quasi-ordinary singularity, the discriminant locus of the projection $\pi$ is contained in the coordinate hyperplanes, the function $\delta(z)=z_{1} \cdots z_{N}$ vanishes there and its Newton polyhedron has just one cone which is the nonnegative orthant. The result is then a consequence of the theorem.

Remark 7.4. Let $\mathcal{A} \subset \mathbb{C}^{N+M}$ and $\pi$ be as in Theorem [7.1] and suppose that $\mathcal{A}$ is a surface (i.e., $N=2$ ). The discriminant locus of $\pi$ is contained in an analytic curve $\delta \subset \mathbb{C}^{2}$. Performing the quadratic transformations needed to solve $\delta$ to both $\delta$ and $\mathcal{A}$ we arrive at a new surface $\tilde{\mathcal{A}}$ and a projection $\tilde{\pi}$ whose discriminant locus $\tilde{\delta}$ has only normal crossings. This fact is used (see [12, 18]) to get a resolution of $\mathcal{A}$. In [3, Chapt. 2] it is shown that the transform of a $\sigma$-wedge is a tubular neighborhood of a connected piece of the exceptional divisor of $\tilde{\delta}$.

\section{REFERENCES}

1. S. S. Abhyankar, On the ramification of algebraic functions, Amer. J. Math. 77 (1955), 575592. MR 17:193c

2. S. S. Abhyankar, Local Analytic Geometry, Academic Press, New York, 1964. MR 31:173

3. F. Aroca, Métodos algebraicos en ecuaciones diferenciales ordinarias en el campo complejo, Tesis Doctoral, Universidad de Valladolid, 2000.

4. F. Aroca and J. Cano, Formal solutions of linear PDEs and convex polyhedra, J. Symbolic Computation 32 (2001), 717-737. MR 2003b:35028

5. J. M. Aroca, H. Hironaka, and J. L. Vicente, Desingularization theorems, Memorias de Matemática del Instituto Jorge Juan; no. 30. Madrid: Consejo Superior de Investigaciones Cientificas (1977). MR 80h:32027

6. A. D. Bruno, Local Methods in Nonlinear, Differential Equations, Springer-Verlag, Berlin, Heidelberg, 1989. MR 90c:58150

7. E. M. Chirka, Complex Analytic Sets, Kluwer Academic Publishers, Dordrecht, 1989. MR 92b:32016

8. W. Fulton, Introduction to Toric Varieties, Annals of Mathematics Studies, no. 131, Princeton University Press, Princeton, NJ, 1993. MR 94g:14028

9. I. M. Gelfand, M. M. Kapranov, and A. V. Zelevinsky, Discriminants, Resultants and Multidimensional Determinants, Birkäuser, Boston, 1994. MR 95e:14045

10. P. D. González Pérez, Singularités quasi-ordinaires toriques et polyèdre de Newton du discriminant, Canadian J. Math 52 (2) (2000), 348-368. MR 2001h:14065

11. H. Hironaka, Introduction to the theory of infinitely near singular points, Memorias de Matemática del Instituto Jorge Juan; no. 28. Madrid: Consejo Superior de Investigaciones Científicas, 1974. MR 53:3349

12. H. B. Laufer, Normal two dimensional singularities, Annals of Mathematics Studies, no. 71, Princeton University Press, Princeton, NJ, 1971. MR 47:8904

13. I. Luengo, A new proof of the Jung-Abhyankar theorem, J. Algebra 85 (1983), no. 2, 399-409. MR 85d:13029

14. J. McDonald, Fiber polytopes and fractional power series, Journal of Pure and Applied Algebra 104 (1995), 213-233. MR 97a:52020

15. W. S. Massey, A basic course in algebraic topology, Graduate Texts in Mathematics, no. 127, Springer-Verlag, New York, 1991. MR 92c:55001

16. M. Saito, B. Sturmfels, and N. Takayama, Groebner Deformations of Hypergeometric Differential Equations, Springer-Verlag, Berlin, 2000. MR 2001i:13036

17. N. Steenrod, The Topology of Fiber Bundles, Princeton University Press, Princeton, NJ, 1951. MR 12:522b 
18. R. J. Walker, Reduction of singularities of an algebraic surface, Ann. Math. (2) 36 (1935), 336-365.

19. M. A. Zurro, The Abhyankar-Jung theorem revisited, Journal of Pure and Applied Algebra 90 (1993), 275-282. MR 94m:13028

Instituto de Matematicas Unam (Unidad Cuernavaca), Apartado Postal 273-3, Administración de Correos 3, CP 62251, Cuernavaca, Morelos, Mexico

E-mail address: fuen@matcuer.unam.mx

Current address: Departamento de Matemática, Instituto de Ciências Matemáticas e de Computação, Universidade de São Paulo, Caixa Postal 668, 13560-970 São Carlos SP, Brazil

E-mail address: fuen@icmc.usp.br 